PHYSICAL REVIEW E 71, 061906 (2005)

\title{
Non-Markovian stochastic resonance: Three-state model of ion channel gating
}

\author{
Igor Goychuk and Peter Hänggi \\ Institute of Physics, University of Augsburg, Universitätsstrasse 1, D-86135 Augsburg, Germany \\ Jose L. Vega and Salvador Miret-Artés \\ Instituto de Matemáticas y Física Fundamental (IMAFF), Consejo Superior de Investigaciones Científicas, Serrano 123,28006 Madrid, \\ Spain \\ (Received 22 February 2005; published 16 June 2005)
}

\begin{abstract}
Stochastic resonance in single voltage-dependent ion channels is investigated within a three-state nonMarkovian modeling of the ion channel conformational dynamics. In contrast to a two-state description one assumes the presence of an additional closed state for the ion channel which mimics the manifold of voltageindependent closed subconformations (inactivated "state"). The conformational transition into the open state occurs through a domain of voltage-dependent closed subconformations (closed "state"). At distinct variance with the standard two-state and also the three-state Markovian approach, the inactivated state is characterized by a broad, nonexponential probability distribution of corresponding residence times. The linear response to a periodic voltage signal is determined for arbitrary distributions of the channel's recovery times. Analytical results are obtained for the spectral amplification of the applied signal and the corresponding signal-to-noise ratio. Alternatively, these results are also derived by use of a corresponding two-state non-Markovian theory which is based on driven integral renewal equations [I. Goychuk and P. Hänggi, Phys. Rev. E 69, 021104 (2004)]. The non-Markovian features of stochastic resonance are studied for a power law distribution of the residence time intervals in the inactivated state which exhibits a large variance. A comparison with the case of biexponentially distributed residence times possessing the same mean value, i.e., the simplest non-Markovian two-state description, is also presented.
\end{abstract}

DOI: 10.1103/PhysRevE.71.061906

PACS number(s): 87.16.Uv, 05.40.-a, 87.10.+e, 87.15.Ya

\section{INTRODUCTION}

Stochastic resonance (SR) [1,2] is by now a wellestablished phenomenon with wide spread applications in physics, chemistry, engineering sciences, and the life sciences. It refers to the fact that in nonlinear stochastic systems an optimal level of applied or intrinsic noise can dramatically boost the response (or, more generally, the transport) to typically weak, time-dependent input signals. This fact plays a prominent role in biology with its abundance of a variety of thresholdlike systems that are subjected to noise influences [1-4]. SR is often also characterized in terms of an underlying stochastic synchronization between an applied stimulus and an intrinsically stochastic dynamics $[1,2,5]$. From the viewpoint of physical biology, the phenomenon of SR in biological sensory systems is commonly assumed to be rooted in the properties of excitable membranes. This being so, it ultimately can be explained in terms of a driven stochastic dynamics of assemblies of ion channels [6-13]. Although biologically relevant SR is generally a property of a cooperative coupling among ion channels [11-13], the study of stochastic resonance in single ion channels carries merit on its own: (1) SR has not yet been convincingly demonstrated on the level of single molecules, with biological ion channels being such proper candidates [7]; (2) ion channels can serve as suitable single-molecular sensors to be utilized in nanodevices.

All these facts in turn have stimulated a vivid interest on this subject matter. The recordings of ion current flowing through a single ion channel, as obtained from a typical patch clamp experiment $[14,15]$, bears close similarity with a simple, driven bistable dynamics $[1,2,16]$. The occurrence of SR in single ion channels thus seems very likely. In reality, the situation is, however, more complex, because (i) the dependence of the opening and closing rates on the voltage and temperature is generally not Arrhenius-like [17-20], a characteristic feature which plays a central role for the occurrence, or nonoccurrence of the SR [9], and (ii) detailed studies of the statistics of the ion current switching events reveal that the probability distributions of the residence time intervals in different conductance states are normally not single exponential. This implies that the observed dynamics of "onoff" conductance fluctuations is generically non-Markovian within a two-state stochastic description [21,22]. In the simplest nontrivial case of a biexponential distribution of the residence time intervals dwelling in the nonconducting conformation, the emerging non-Markovian dynamics can be embedded into a three-state Markovian dynamics [15] with an extra (unobservable) closed state. The presence of such a third state (or, more generally, a number of additional substates) can, however, be inferred from the bursting character of the observed dynamics when the ion channel dynamics after switching between its open and closed states for a number of times suddenly stops, and then revives again later after a notably longer time span has elapsed (as compared with the typical sojourn length in the open or closed state). The presence of such a third state can be considered as a closed inactivated state. This "third" state in fact does not constitute a single state, but rather a manifold of many substates. As a consequence, the recovery of the ion channel from its inactive state does not present an exponential rate process, but will be governed by a nonexponential distribution of corre- 


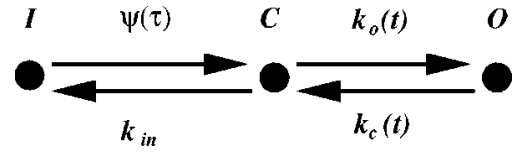

FIG. 1. Sketch of the three-state model setup with generally time-dependent opening and closing rates and with a nonexponential residence time statistics in the inactivated state.

sponding residence times. This feature, i.e., the absence of a well-defined time scale for recovery from inactivation, can be observed in various ion channels [23].

Our main objective here is to investigate the basic features of SR occurring in single ion channels within the framework of a three-state non-Markovian dynamics. In addition, we contrast this approach with the two-state nonMarkovian theory of stochastic resonance developed in prior works $[24,25]$.

\section{MODEL SETUP}

Following the reasoning put forward in Refs. $[19,20]$ we consider a discrete state model of ion channel gating, whose essentials are depicted in Fig. 1. It consists of three "states": an open one $(O)$, a closed one $(C)$, and an inactivated state $(I)$. The inactivated state $I$ is assumed to correspond to the manifold of voltage-insensitive conformations of the ion channel protein and the opening $C \rightarrow O$ and closing $O \rightarrow C$ transitions are associated with the motion of a voltage sensor and the opening or closing of the channel's gate. The stochastic transitions between the open and closed states are characterized by generally time-dependent opening and closing rates, $k_{o}(t)$ and $k_{c}(t)$, respectively. These rates are assumed to depend on an externally applied, time-dependent voltage signal.

In the spirit of the modeling put forward in [19,20,26-29] we assume that inactivation occurs from the closed state of the ion channel. In doing so, we are dealing with an archetype model of gating with opening, closing, and inactivation dynamics [14]. Furthermore, unlike in the standard Markovian modeling [14,15], we assume that the transition from the inactivated, voltage-independent state to the closed state is not rate limited, but rather is characterized by a broad distribution of rates. Put differently, we model the step from inactivation toward the closing state (cf. Fig. 1) by a nonexponential distribution $\psi(\tau)$ of the residence time intervals spent in the inactivated state. This distribution will be assumed to possess a finite average $\left\langle\tau_{r}\right\rangle:=\int_{0}^{\infty} \tau \psi(\tau) d \tau$. It is voltage independent but arbitrary otherwise. Furthermore, it is assumed that the channel's inactivation occurs from the closed state with a voltage-independent rate $k_{i n}$. The voltage independence of $I \leftrightarrow C$ transitions follows from the underlying character of the conformational dynamics: namely, one assumes that the $I \leftrightarrow C$ transitions occur in a direction transverse to the direction of $C \leftrightarrow O$ transitions [20]. These latter transitions are bound to the relocation of the gating charge across the membrane, while the former transitions are not related to a charge redistribution; for further details we refer the reader to the discussion in Ref. [20].
This chosen distribution of rates accounts for the fact that for several types of ion channels the recovery process does not have a well-defined time scale [23]. This circumstance in turn implies that the gating process is non-Markovian within our three-state description. The standard Markovian threestate description is recovered when $\psi(\tau)=k_{r} \exp \left(-k_{r} \tau\right)$, where $k_{r}:=\left\langle\tau_{r}\right\rangle^{-1}$ is the rate of the $I \rightarrow C$ transition.

\section{NON-MARKOV THEORY OF THREE-STATE GATING}

The time evolution for the probabilities to occupy the open, closed, or inactivated state, $p_{o}(t), p_{c}(t)$, and $p_{I}(t)$, respectively, is governed by the following generalized master equations:

$$
\dot{p}_{o}(t)=k_{o}(t) p_{c}(t)-k_{c}(t) p_{o}(t)
$$

$$
\dot{p}_{c}(t)=-\left[k_{i n}+k_{o}(t)\right] p_{c}(t)+k_{c}(t) p_{o}(t)+\int_{t_{0}}^{t} \Gamma\left(t-t^{\prime}\right) p_{I}\left(t^{\prime}\right) d t^{\prime}
$$

$$
\dot{p}_{I}(t)=k_{i n} p_{c}(t)-\int_{t_{0}}^{t} \Gamma\left(t-t^{\prime}\right) p_{I}\left(t^{\prime}\right) d t^{\prime} .
$$

The Laplace-transformed kernel $\Gamma(t)$ reads

$$
\widetilde{\Gamma}(s)=\frac{s \widetilde{\psi}(s)}{1-\widetilde{\psi}(s)},
$$

with $\widetilde{\psi}(s)$ denoting the Laplace transform of $\psi(\tau)$ (the tilde denotes throughout the Laplace transform of the corresponding function). The solution depends also on the initial condition, chosen for example to read $p_{c}\left(t_{0}\right)=1$, at initial time $t$ $=t_{0}$.

This set of equations can formally be derived following the approaches in Refs. [25,30-33]. Alternatively, this very set of non-Markovian evolution equations can be obtained more directly as well. The terms not expected from naive grounds in Eq. (1) are the ones that involve memory. Let us assume that the ion channel is prepared in the state $I, p_{I}(0)$ $=1$ at $t_{0}=0$, and impose $k_{i n} \rightarrow 0$, i.e., no returns are possible. Then, the leakage of probability $p_{I}(t)$ is due to the transition into the state $C$, i.e., $p_{I}(t)$ must equal (with such an absorbing boundary condition) the survival probability $\Phi(t)$ $=\int_{t}^{\infty} \psi(\tau) d \tau$. With this leakage given by $\dot{\Phi}(t)=-\int_{0}^{t} \Gamma(t$ $\left.-t^{\prime}\right) \Phi\left(t^{\prime}\right) d t^{\prime}$ we readily find by use of the Laplace transform method the relation in Eq. (2). The description in Eq. (1) portrays a driven (i.e., inhomogeneous in time) three-state, non-Markovian renewal process with the corresponding residence time densities (RTDs) [25,34], reading

$$
\begin{gathered}
\psi_{o}(t+\tau, t)=k_{c}(t+\tau) \exp \left(-\int_{t}^{t+\tau} k_{c}\left(t^{\prime}\right) d t^{\prime}\right), \\
\psi_{c}(t+\tau, t)=\left[k_{i n}+k_{o}(t+\tau)\right] \exp \left(-\int_{t}^{t+\tau}\left[k_{i n}+k_{o}\left(t^{\prime}\right)\right] d t^{\prime}\right),
\end{gathered}
$$




$$
\psi_{I}(\tau)=\psi(\tau)
$$

Note in particular that $\psi_{o}(t+\tau, t)$ and $\psi_{c}(t+\tau, t)$ depend not only on the length of residence time intervals $\tau$, but also on the entrance time point $t$. We refer the reader to Ref. [25] for a detailed trajectory description of such driven renewal processes. These conditioned, nonstationary two-time RTDs $\psi_{j}(\tau \mid t):=\psi_{j}(t+\tau, t)(j=o, c)$ are given as the negative time derivatives of the corresponding survival probabilities $\Phi_{j}(\tau \mid t):=\Phi_{j}(t+\tau, t)$; i.e., $\psi_{j}(\tau \mid t):=-d \Phi_{j}(\tau \mid t) / d \tau$.

The averaged, time-dependent conductance $g(t)$ of the considered ion channel reads $\langle g(t)\rangle=p_{o}(t) g_{o}+\left[p_{c}(t)\right.$ $\left.+p_{I}(t)\right] g_{c}$, where $g_{o}$ and $g_{c}$ are the conductances of the open and closed conformations, respectively. In the absence of a time-dependent signal, $\langle g(t)\rangle=\langle g\rangle_{s t}=p_{o}^{s t} g_{o}+\left(p_{c}^{s t}+p_{I}^{s t}\right) g_{c}$, where $p_{\alpha}^{s t}$ with $\alpha=o, c, I$ is the stationary solution of Eq. (1) in the absence of driving. For the following we assume a periodic voltage signal given by

$$
V_{s}(t)=A \cos (\Omega t) \text {. }
$$

The mean deviation of the channel conductance $\langle\delta g(t)\rangle:=\langle g(t)\rangle-\langle g\rangle_{s t}$ from its stationary value thus reads at asymptotic times within a linear response approximation [1],

$$
\langle\delta g(t)\rangle=A|\tilde{\chi}(\Omega)| \cos [\Omega t-\varphi(\Omega)],
$$

where $\varphi(\Omega)$ is the corresponding phase shift [1,29]. In Eq. (5), $\tilde{\chi}(\Omega)$ denotes the linear response function in the frequency domain. The linear response result for the spectral amplification (SPA) of the signal $\eta(\Omega)$ is given by $\eta(\Omega)$ $=|\widetilde{\chi}(\Omega)|^{2}[1]$.

\section{A. Linear response theory}

In order to evaluate the linear response function we expand the solution of Eq. (1) as $p_{c}(t)=\sum_{k=-\infty}^{\infty} r_{k}(t) \exp (-i k \Omega t)$ and $p_{o}(t)=\sum_{k=-\infty}^{\infty} q_{k}(t) \exp (-i k \Omega t)$. The solution for $p_{I}(t)$ follows by virtue of probability conservation, i.e., $p_{I}(t)=1$ $-p_{o}(t)-p_{c}(t)$. In the limit $t \rightarrow \infty$ the solutions become time periodic $[1,16]$ with time-independent coefficients $r_{k}$ and $q_{k}$ which depend on the amplitude strength $A$ and frequency $\Omega$. The asymptotic, nonlinear periodic solution thus reads

$$
\begin{aligned}
& p_{c}^{a s}(t)=\sum_{k=-\infty}^{\infty} r_{k} \exp (-i k \Omega t), \quad r_{-k}=r_{k}^{*}, \\
& p_{o}^{a s}(t)=\sum_{k=-\infty}^{\infty} q_{k} \exp (-i k \Omega t), \quad q_{-k}=q_{k}^{*} .
\end{aligned}
$$

The linear response function $\tilde{\chi}(\Omega)$ is encoded in the first Fourier term [29]. It reads

$$
\tilde{\chi}(\Omega)=2 \Delta g \lim _{A \rightarrow 0} \frac{q_{1}}{A},
$$

where $\Delta g:=g_{o}-g_{c}$ is the difference of ion channel conductances in the open and closed states, respectively.

To start out, we assume the following small-signal expansion of the time-dependent reaction rates:

$$
k_{o, c}(t)=\nu_{0} \exp \left[-\Delta G_{o, c}(V(t)) / k_{B} T\right] \approx k_{o, c}^{(0)}\left[1-\beta_{o, c} V_{s}(t)\right],
$$

where in the absence of driving

$$
k_{o, c}^{(0)}=\nu_{0} \exp \left[-\Delta G_{o, c}\left(V_{0}\right) / k_{B} T\right],
$$

with $\Delta G_{o, c}\left(V_{0}\right)$ denoting the corresponding static free energy barriers, $V_{0}$ being a static voltage in the absence of signal, i.e., $V(t)=V_{0}+V_{s}(t)$, and $\beta_{o, c}=-\left.\left[\left(d \ln k_{o, c}^{(0)}\right) / d V\right]\right|_{V=V_{0}}$.

The substitution of Eq. (6) into Eq. (1) and taking Eqs. (4) and (8) into account yields for $t_{0} \rightarrow-\infty$ (this procedure is equivalent to taking $t_{0}=0$ and $t \rightarrow \infty$ ) the following recurrence relations:

$$
\begin{gathered}
{[-i k \Omega+\widetilde{\Gamma}(-i k \Omega)]\left(r_{k}+q_{k}\right)=-k_{i n} r_{k}+\frac{1}{\left\langle\tau_{r}\right\rangle} \delta_{k, 0},} \\
\left(-i k \Omega+k_{c}^{(0)}\right) q_{k}+k_{c}^{(1)}\left(q_{k-1}+q_{k+1}\right)=k_{o}^{(0)} r_{k}+k_{o}^{(1)}\left(r_{k-1}+r_{k+1}\right),
\end{gathered}
$$

where

$$
k_{o, c}^{(1)}=-\frac{1}{2} A k_{o, c}^{(0)} \beta_{o, c} .
$$

In Eq. (10), we used that $\lim _{s \rightarrow 0} \widetilde{\Gamma}(s)=1 /\left\langle\tau_{r}\right\rangle$ which follows from the expansion $\tilde{\psi}(s)=1-s\left\langle\tau_{r}\right\rangle+o(s)$, where $o(s)$ stands for the terms such that $\lim _{s \rightarrow 0}[o(s) / s]=0$. From the first equation in (10) we obtain

$$
q_{0}=1-\left(1+k_{\text {in }}\left\langle\tau_{r}\right\rangle\right) r_{0}
$$

for $k=0$ and

$$
q_{k}=-\left(1+\frac{k_{i n}}{-i k \Omega+\widetilde{\Gamma}(-i k \Omega)}\right) r_{k}
$$

otherwise. Therefore, either $q_{k}$ or $r_{k}$ can be determined from the second equation in Eq. (10) which thus uncouples into a recurrence relation for either $q_{k}$ or $r_{k}$. To the lowest order in $A, q_{0}=q_{0}^{(0)}+O\left(A^{2}\right)$ and $r_{0}=r_{0}^{(0)}+O\left(A^{2}\right)$ where $\left\{q_{0}^{(0)}, r_{0}^{(0)}\right\}$ are the values when the perturbation is absent. A term linear in $A$ is absent because a change from $A$ to $-A$ (which is equivalent to a phase shift by $\pi$ ) cannot result in a shift of steady state populations $q_{0}$ and $r_{0}$. Moreover, $q_{1} \propto A$ and $r_{1} \propto A$ to leading order in $A$. Likewise, the expansions of $q_{2}$ and $r_{2}$ in $A$ start out from $A^{2}$.

Then, by taking into account $r_{0}=k_{c}^{(0)} q_{0} / k_{o}^{(0)}+O\left(A^{2}\right)$ in Eqs. (10), (12), and (13) we obtain after some algebraic manipulations

$$
\begin{aligned}
& r_{0}=\frac{k_{c}^{(0)}}{k_{o}^{(0)}+k_{c}^{(0)}\left(1+k_{i n}\left\langle\tau_{r}\right\rangle\right)}+O\left(A^{2}\right), \\
& q_{0}=\frac{k_{o}^{(0)}}{k_{o}^{(0)}+k_{c}^{(0)}\left(1+k_{i n}\left\langle\tau_{r}\right\rangle\right)}+O\left(A^{2}\right),
\end{aligned}
$$

and 


$$
\begin{aligned}
q_{1}= & \frac{1}{2} A \frac{\beta_{c}-\beta_{o}}{\left\langle\tau_{o}\right\rangle+\left\langle\tau_{c^{\prime}}\right\rangle} \frac{1}{k_{c}^{(0)}-i \Omega+i \Omega k_{o}^{(0)} /\left\{i \Omega-k_{i n}[1-\tilde{\psi}(-i \Omega)]\right\}} \\
& +O\left(A^{2}\right),
\end{aligned}
$$

where

$$
\left\langle\tau_{o}\right\rangle=1 / k_{c}^{(0)}
$$

is the mean residence time in the open state. Furthermore,

$$
\left\langle\tau_{c^{\prime}}\right\rangle=\left(1+k_{i n}\left\langle\tau_{r}\right\rangle\right) / k_{o}^{(0)}
$$

is the mean residence time within the set of closed states $c^{\prime}=(C, I)$ in the absence of time-dependent driving.

This latter quantity is defined as $\left\langle\tau_{c^{\prime}}\right\rangle:=\int_{0}^{\infty} \tau \psi_{c^{\prime}}(\tau) d \tau$, where $\psi_{c^{\prime}}(\tau)$ is the stationary RTD in the set of compound closed states, when no time-dependent signal acts, i.e., $V_{s}(t)=0$. This auxiliary quantity is obtained as follows. The channel is prepared in the closed state $C$ at $t_{0}=0$, i.e., $p_{c}(0)$ $=1$, and the back transition $O \rightarrow C$ is set to zero by imposing $k_{c} \rightarrow 0$. Then, the solution of the first two equations in (1) in the absence of driving yields the stationary survival probability of the compound closed states as $\Phi_{c^{\prime}}(t)=p_{c}(t)+p_{I}(t)$ and $\psi_{c^{\prime}}(t)$ follows as $\psi_{c^{\prime}}(\tau)=-d \Phi_{c^{\prime}}(\tau) / d \tau$. Using this scheme we find

$$
\widetilde{\Phi}_{c^{\prime}}(s)=\frac{1+k_{i n} /[s+\widetilde{\Gamma}(s)]}{s+k_{o}^{(0)}+k_{i n} s /[s+\widetilde{\Gamma}(s)]}
$$

and in virtue of $\widetilde{\psi}_{c^{\prime}}(s)=1-s \widetilde{\Phi}_{c^{\prime}}(s)$

$$
\tilde{\psi}_{c^{\prime}}(s)=\frac{k_{o}^{(0)}}{s+k_{o}^{(0)}+k_{i n} s /[s+\widetilde{\Gamma}(s)]} .
$$

By use of Eq. (2) in Eq. (19) this stationary distribution of the residence times can be recast as

$$
\tilde{\psi}_{c^{\prime}}(s)=\frac{k_{o}^{(0)}}{s+k_{o}^{(0)}+k_{i n}[1-\widetilde{\psi}(s)]} .
$$

Furthermore, $\left\langle\tau_{c^{\prime}}\right\rangle$ in Eq. (17) follows from Eq. (18) as $\left\langle\tau_{c^{\prime}}\right\rangle=\widetilde{\Phi}_{c^{\prime}}(0)$.

Let us introduce also the auxiliary function

$$
\tilde{\mathcal{G}}(s)=\frac{\left[1-\tilde{\psi}_{o}(s)\right]\left[1-\tilde{\psi}_{c^{\prime}}(s)\right]}{1-\tilde{\psi}_{o}(s) \tilde{\psi}_{c^{\prime}}(s)},
$$

where $\tilde{\psi}_{o}(s)=k_{c}^{(0)} /\left(s+k_{c}^{(0)}\right)$ is the Laplace transform of the stationary RTD of the open time intervals. Then, Eq. (15) can be rewritten in a more compact form as

$$
q_{1}=\frac{1}{2} A \frac{\beta_{c}-\beta_{o}}{\left\langle\tau_{o}\right\rangle+\left\langle\tau_{c^{\prime}}\right\rangle} \frac{\tilde{\mathcal{G}}(-i \Omega)}{-i \Omega}+O\left(A^{2}\right) .
$$

Since $k_{i n}\left\langle\tau_{r}\right\rangle$ does not depend on voltage $V$, $\beta_{o^{\prime}}:=\left.\left[\left(d \ln \left\langle\tau_{c^{\prime}}\right\rangle\right) / d V\right]\right|_{V=V_{0}}=-\left.\left[\left(d \ln k_{o}^{(0)}\right) / d V\right]\right|_{V=V_{0}}=\beta_{o}$ and the result in Eq. (22) coincides with the result of the phenomenological two-state theory of non-Markovian SR put forward in $[24,25]$. Upon combining with Eq. (7), Eq. (22) yields the linear response result

$$
\widetilde{\chi}(\Omega)=\frac{\left(\beta_{c}-\beta_{o}\right) \Delta g}{\left\langle\tau_{o}\right\rangle+\left\langle\tau_{c^{\prime}}\right\rangle} \frac{\widetilde{\mathcal{G}}(-i \Omega)}{-i \Omega},
$$

which in fact coincides with Eq. (61) in Ref. [25].

The just outlined procedure can as well be extended into the nonlinear response regime to obtain nonlinear response functions of required order in the signal amplitude. This, however, is beyond the scope of this work. This central result in Eq. (23) is alternatively derived in the Appendix by making use of the two-state non-Markovian theory detailed in Ref. [25].

\section{B. Explicit results in terms of thermodynamic free energies}

We next introduce formally the effective free energy bias $\epsilon(T)$, i.e.,

$$
\frac{\left\langle\tau_{o}\right\rangle}{\left\langle\tau_{c^{\prime}}\right\rangle}=\exp \left(-\frac{\epsilon(T)}{k_{B} T}\right) .
$$

In accordance with the relations (16) and (17) we obtain

$$
\epsilon(T)=\Delta G_{o}\left(V_{0}\right)-\Delta G_{c}\left(V_{0}\right)+k_{B} T \ln \left(1+k_{i n}\left\langle\tau_{r}\right\rangle\right) .
$$

Using that $\Delta G_{o}\left(V_{0}\right)=G^{\#}-G_{c}$ and $\Delta G_{c}\left(V_{0}\right)=G^{\#}-G_{o}$, where $G^{\#}$ is the (Gibbs) free energy of the transition state, and $G_{o, c}=H_{o, c}-T S_{o, c}$ is the free energy of the open (closed) conformation, Eq. (25) can be recast as

$$
\epsilon(T)=\Delta H-T \Delta S+k_{B} T \ln \left(1+k_{i n}\left\langle\tau_{r}\right\rangle\right),
$$

where $\Delta H:=H_{o}-H_{c}$ is the difference of thermodynamic enthalpies of the open and closed conformations (or of the internal energies, if no volume change of the macromolecule occurs at the conformational transition) and $\Delta S:=S_{o}-S_{c}$ is the corresponding entropy difference.

Assuming that the free energy barriers in Eq. (8) have linear dependence of voltage, $\Delta G_{o}(V)=\Delta G_{o}-q(1-\delta) V$ and $\Delta G_{c}(V)=\Delta G_{c}+q \delta V$, where $q$ is the gating charge and $0<\delta$ $<1$ is a constant $[14,20]$, we find that $\beta_{c}-\beta_{o}=-q /\left(k_{B} T\right)$. For the spectral amplification of the conductance response $\eta$ $=|\widetilde{\chi}(\Omega)|^{2}$ we obtain then from Eq. (23) our first main result

$$
\eta(\Omega, T)=\frac{(\Delta g)^{2} q^{2}}{16\left(k_{B} T\right)^{2}} \frac{\nu^{2}(T)}{\cosh ^{4}\left[\epsilon(T) / 2 k_{B} T\right]} \frac{|\widetilde{\mathcal{G}}(-i \Omega)|^{2}}{\Omega^{2}},
$$

with $\epsilon(T)$ given in Eq. (25), while $\nu(T)=\left\langle\tau_{o}\right\rangle^{-1}+\left\langle\tau_{c^{\prime}}\right\rangle^{-1}$ denotes the sum of the closing and opening rates. $\widetilde{\mathcal{G}}(s)$ in Eqs. (21) and (27) reads explicitly

$$
\widetilde{\mathcal{G}}(s)=\frac{s}{s+k_{c}^{(0)}+s k_{o}^{(0)} /\left\{s+k_{\text {in }}[1-\widetilde{\psi}(s)]\right\}},
$$

where $k_{c}^{(0)}, k_{o}^{(0)}, k_{\text {in }}$ are the unperturbed rates and $\psi(\tau)$ is the residence time distribution. The result in Eq. (27) coincides, apart from a model specific constant, with the expression given in Ref. [24], Eq. (25) therein. Within the linear response approximation the corresponding signal-to-noise ratio 
(SNR) at the angular driving frequency $\Omega$ is obtained from $R_{S N}(\Omega, T):=\pi A^{2} \eta / S_{N}(\omega=\Omega)$, where $S_{N}(\omega)$ denotes the spectral power density of conductance fluctuations in the absence of signal $V_{s}(t)$. By use of the Stratonovich formula for the autocorrelation function of the alternating renewal process [35] and the Wiener-Khinchin theorem one finds for $S_{N}(\omega)$ $[25,35]$

$$
S_{N}(\omega)=\frac{2(\Delta g)^{2}}{\left\langle\tau_{o}\right\rangle+\left\langle\tau_{c^{\prime}}\right\rangle} \frac{1}{\omega^{2}} \operatorname{Re}[\tilde{\mathcal{G}}(i \omega)]
$$

Therefore, the SNR equals the result derived in Refs. [24,25], namely,

$$
\mathrm{R}_{S N}(\Omega, T)=\frac{\pi A^{2} q^{2}}{8\left(k_{B} T\right)^{2}} \frac{\nu(T)}{\cosh ^{2}\left[\epsilon(T) / 2 k_{B} T\right]} N(\Omega),
$$

where

$$
N(\Omega)=\frac{|\widetilde{\mathcal{G}}(i \Omega)|^{2}}{\operatorname{Re}[\widetilde{\mathcal{G}}(i \Omega)]}
$$

provides the specific function which accounts for manifest non-Markovian effects. In the low frequency limit $\Omega \rightarrow 0$, $N(\Omega)$ approaches the limit $N(0)=2 /\left(C_{o}^{2}+C_{c^{\prime}}^{2}\right)[24,25]$, where $C_{o}$ and $C_{c^{\prime}}$ are the coefficients of variation of the RTDs of the open and compound closed states, respectively.

By use of the expansion $\widetilde{\psi}(s)=1-s\left\langle\tau_{r}\right\rangle+o(s)$ in Eq. (28) and Eq. (17) we find in the adiabatic limit $\Omega \rightarrow 0$ that the spectral power amplification acquires the universal form, reading

$$
\eta(\Omega \rightarrow 0, T)=\frac{(\Delta g)^{2} q^{2}}{16\left(k_{B} T\right)^{2}} \frac{1}{\cosh ^{4}\left[\epsilon(T) / 2 k_{B} T\right]} .
$$

This result holds in the presence of asymmetry with nonvanishing $\epsilon(T)$. In such a case, the SPA (32) can exhibit a sharp stochastic resonance at the physiologically relevant temperatures when $\epsilon(T)$ changes sign, when the probabilities of the channel to be open or to stay closed become equal. This corresponds to the opening threshold for the case detailed below of an ion channel sensitive to cold (or, vice versa, sensitive to heat, if the open state is preferred from an entropic viewpoint). The cold-sensitive or heat-sensitive ion channels [36] present appropriate candidates to reveal this entropic SR effect. This feature at very small driving frequencies, which mimics a Markovian behavior, has thus nothing to do with non-Markovian properties; it is solely due to an entropic asymmetry. The non-Markovian effects emerge, however, at small, but finite frequencies in the corresponding SPA curves (see in Ref. [24]). Most prominent non-Markovian, long-time memory effects distinctly suppress, however, the SNR in the low frequency domain $[24,25]$. For $\Omega \rightarrow 0$, we obtain (with $C_{o}=1$ )

$$
\mathrm{R}_{S N}(\Omega \rightarrow 0, T)=\frac{\pi A^{2} q^{2}}{8\left(k_{B} T\right)^{2}} \frac{\nu(T)}{\cosh ^{2}\left[\epsilon(T) / 2 k_{B} T\right]} \frac{2}{1+C_{c^{\prime}}^{2}},
$$

with $C_{c^{\prime}}>1$. Thus, the $R_{S N}(\Omega=0, T)$ is fully suppressed, making the detection of low frequency signals barely possible, e.g., for the power law distribution $\psi(\tau) \propto 1 / \tau^{2+\alpha}$ with $0<\alpha<1$ considered below. This suppression occurs due to the $1 / f^{1-\alpha}$ noise feature in the spectral power $S_{N}(\Omega)$ for small frequencies. Let us illustrate now these general considerations with two particular models.

\section{ION CHANNEL GATING: BIEXPONENTIAL DISTRIBUTION VERSUS A POWER LAW}

The current three-state model provides a suitable framework to clarify the role of power law distributed residence times in the inactivated and closed states as compared with the simplest two-state non-Markovian situation (with respect to the observable dynamics) of a biexponential distribution, which, likewise, can be embedded into a three-state Markovian description.

\section{A. The case of a biexponential distribution}

We start our driven channel gating investigation with the simplest case of an exponential residence time distribution of the transition $I \rightarrow C$, i.e., $\psi(\tau)=k_{r} \exp \left(-k_{r} \tau\right)$. Then, Eq. (20) yields

$$
\tilde{\psi}_{c^{\prime}}(s)=\frac{k_{o}^{(0)}\left(s+k_{r}\right)}{\left(s+k_{o}^{(0)}\right)\left(s+k_{r}\right)+k_{i n} s} .
$$

The inversion of Eq. (34) yields a biexponential probability density, i.e.,

$$
\psi_{c^{\prime}}(\tau)=c_{1} \lambda_{1} \exp \left(-\lambda_{1} \tau\right)+c_{2} \lambda_{2} \exp \left(-\lambda_{2} \tau\right)
$$

with the rate coefficients $\lambda_{1,2}=\frac{1}{2}\left(k_{o}^{(0)}+k_{\text {in }}\right.$ $+k_{r} \pm \sqrt{\left.\left(k_{o}^{(0)}-k_{i n}-k_{r}\right)^{2}+4 k_{o}^{(0)} k_{i n}\right)}$ and corresponding weight factors $\quad c_{1,2}=\frac{1}{2}\left[1 \pm\left(k_{o}^{(0)}-k_{i n}-k_{r}\right) / \sqrt{\left(k_{o}^{(0)}-k_{i n}-k_{r}\right)^{2}+4 k_{o}^{(0)} k_{i n}}\right]$. The mean residence time corresponding to this RTD (35) is

$$
\left\langle\tau_{c^{\prime}}\right\rangle=\frac{1}{k_{o}^{(0)}}\left(1+\frac{k_{i n}}{k_{r}}\right) .
$$

Such a nonexponential, i.e., biexponential, RTD can actually be very broad as characterized by the corresponding coefficient of variation $C:=\sqrt{\left\langle\tau^{2}\right\rangle-\langle\tau\rangle^{2}} /\langle\tau\rangle$, yielding from Eq. (35)

$$
C_{c^{\prime}}=\sqrt{1+\frac{2 k_{o}^{(0)} k_{i n}}{\left(k_{\text {in }}+k_{r}\right)^{2}}} .
$$

Indeed, for $k_{r} \ll k_{\text {in }} \ll k_{o}^{(0)}$ we deduce from Eq. (37) that $C_{c^{\prime}}$ $\approx \sqrt{2 k_{o}^{(0)} / k_{i n}} \gg 1$. This implies that the distribution (35) has a small, but very broad long-time tail, which in turn results in a large variance of the residence times. This finding carries important consequences: As shown in Refs. [24,25] the signal-to-noise ratio $R_{S N}$ is then strongly suppressed in the low-frequency limit $\Omega \rightarrow 0$ by the factor $1 / N(0)=\frac{1}{2}\left(C_{o}^{2}\right.$ 
$\left.+C_{c^{\prime}}^{2}\right)$, where $C_{o}$ is the coefficient of variation for $\psi_{o}(\tau)$, $C_{o}=1$ in the present case. This presents a first manifest nonMarkovian effect which is present already within this simplest non-Markovian setting. We also note that the auxiliary function $\widetilde{\mathcal{G}}(s)$ can be recast as

$$
\widetilde{\mathcal{G}}(s)=\frac{s\left(s+k_{\text {in }}+k_{r}\right)}{\left[s+\mu_{1}(T)\right]\left[s+\mu_{2}(T)\right]},
$$

where

$$
\begin{aligned}
\mu_{1,2}(T)= & \frac{1}{2}\left(k_{o}^{(0)}+k_{c}^{(0)}+k_{\text {in }}\right. \\
& +k_{r} \pm \sqrt{\left.\left(k_{o}^{(0)}+k_{c}^{(0)}-k_{\text {in }}-k_{r}\right)^{2}+4 k_{o}^{(0)} k_{\text {in }}\right)}
\end{aligned}
$$

are the decay rates of the conductance time correlations. By use of Eqs. (38) and (31) the results in Eqs. (27) for the SPA and (30) for the SNR assume explicitly the form

$$
\begin{aligned}
\eta(\Omega, T)= & \frac{(\Delta g)^{2} q^{2}}{16\left(k_{B} T\right)^{2}} \frac{\nu^{2}(T)}{\cosh ^{4}\left[\epsilon(T) / 2 k_{B} T\right]} \\
& \times \frac{\Omega^{2}+\left(k_{\text {in }}+k_{r}\right)^{2}}{\left[\Omega^{2}+\mu_{1}^{2}(T)\right]\left[\Omega^{2}+\mu_{2}^{2}(T)\right]}
\end{aligned}
$$

and

$$
\begin{aligned}
\mathrm{R}_{S N}(\Omega, T)= & \frac{\pi A^{2} q^{2}}{8\left(k_{B} T\right)^{2}} \frac{\nu(T)}{\cosh ^{2}\left[\epsilon(T) / 2 k_{B} T\right]} \\
& \times \frac{\Omega^{2}+\left(k_{i n}+k_{r}\right)^{2}}{\Omega^{2}+\left(k_{\text {in }}+k_{r}\right)^{2}+k_{o}^{(0)} k_{\text {in }}},
\end{aligned}
$$

respectively. The remaining parameters are $\nu(T)=\left\langle\tau_{o}\right\rangle^{-1}$ $+\left\langle\tau_{c^{\prime}}\right\rangle^{-1}=k_{c}^{(0)}+k_{o}^{(0)} k_{r} /\left(k_{r}+k_{i n}\right)$ with $k_{o, c}^{(0)}$ in Eq. (9) and $\epsilon(T)$ $=\Delta G_{o}\left(V_{0}\right)-\Delta G_{c}\left(V_{0}\right)+k_{B} T \ln \left(1+k_{i n} / k_{r}\right) \quad$ with exponential Arrhenius rates $k_{i n}=\nu_{0} \exp \left(-\Delta G_{i n} / k_{B} T\right)$ and $k_{r}=\nu_{0} \exp [$ $\left.-\Delta G_{r} / k_{B} T\right][37]$.

The results in Eqs. (40) and (41) constitute central results for the SR occurring in a three-state Markovian model of gating in ion channels possessing an inactivation from the closed state. At the same time, these results correspond to a simplest non-Markovian two-state model of the observable dynamics of conductance fluctuations.

We performed numerical calculations for the set of test parameters given in Table I which is chosen to mimic the experimental temperature dependence of the cold-sensitive ion channels (see in Ref. [36]).

The temperature dependence of the corresponding Markovian transition rates are given in Fig. 2(a). For this set of parameters, the coefficient of variation of the closed residence time (compound state) within the three-state Markovian description is $C_{c^{\prime}} \approx 17.14$ and the low-frequency SNR is suppressed by the factor of $1 / N(0) \approx 147.43$ as compared with the corresponding two-state Markovian case with the same rates $k_{c}^{(0)}$ and $k_{o^{\prime}}^{(0)}:=\left\langle\tau_{c^{\prime}}\right\rangle^{-1}$. Their temperature dependence is depicted in Fig. 2(b). The frequency prefactor $\nu_{0}$ has been taken to be $\nu_{0}=6.11 \times 10^{12} \mathrm{~s}^{-1}$. This value corresponds approximately to a gas-phase value of $k_{B} T / h$ at $T=20^{\circ} \mathrm{C}$. It clearly overestimates any effects that relate to friction and
TABLE I. Free energy barriers $\Delta G_{\alpha}=\Delta H_{\alpha}-T \Delta S_{\alpha}$.

$\alpha \quad$ Enthalpy part $\Delta H_{\alpha}(\mathrm{kJ} / \mathrm{mol})^{\mathrm{a}} \quad$ Entropy part $\Delta S_{\alpha}$ (units of $k_{B}$ )

\begin{tabular}{lcc}
$c$ & 175 & 52 \\
$o$ & 15 & -10 \\
in & 15 & -15 \\
$r$ & 15 & -20 \\
\hline
\end{tabular}

${ }^{\mathrm{a}}$ Table depicts the corresponding parameters for the free energy barriers that enter the related rate coefficients. Here, $\Delta H_{\alpha}$ must be divided by the Avogadro number $N_{A}$ to obtain the corresponding value per single molecule. For example, $\Delta H_{c}=175 \mathrm{~kJ} / \mathrm{mol}$ thus corresponds (we use the Boltzmann constant $k_{B}$ and not the gas constant $R=k_{B} N_{A}$ in the rate expressions) to a value $\Delta H_{c} \approx 2.91$ $\times 10^{-19} \mathrm{~J} \approx 1.81 \mathrm{eV}$, etc.

other details determining the transmission coefficient $\kappa$ in condensed phases. The correct estimation of this prefactor would require a more elaborate theory of the Kramers type [37] rather than the absolute rate theory used here. Nevertheless, this ambiguity does not play a role if we assume that the frequency prefactor $\nu_{0}$ is one and the same for all the rate
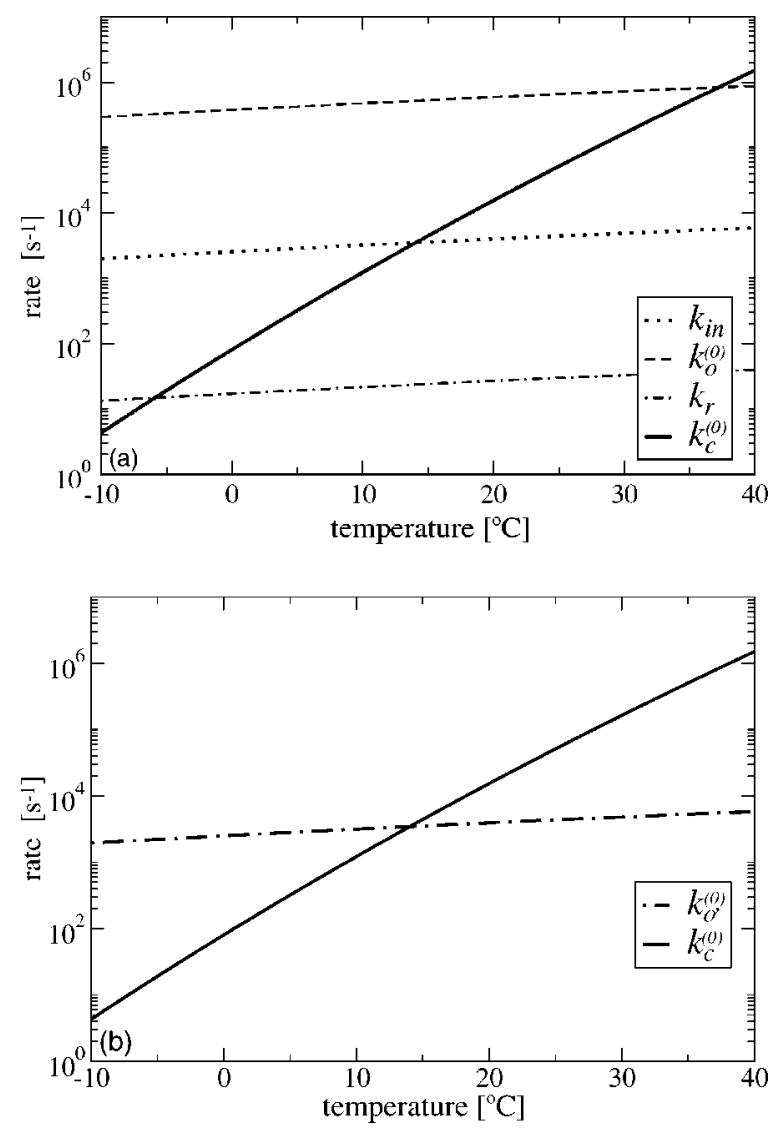

FIG. 2. Temperature dependence of the rates used for the ion channel gating dynamics, absent the driving: (a) the considered three-state Markovian model; (b) the corresponding two-state nonMarkov model with the effective opening rate defined as $k_{o^{\prime}}^{(0)}$ $:=\left\langle\tau_{c^{\prime}}\right\rangle^{-1}$, where $\left\langle\tau_{c^{\prime}}\right\rangle$ is the mean residence time. The rate $k_{c}^{(0)}$ denotes again the transition from the open state $O$ toward the closed state $C$ in the absence of time-dependent driving. 

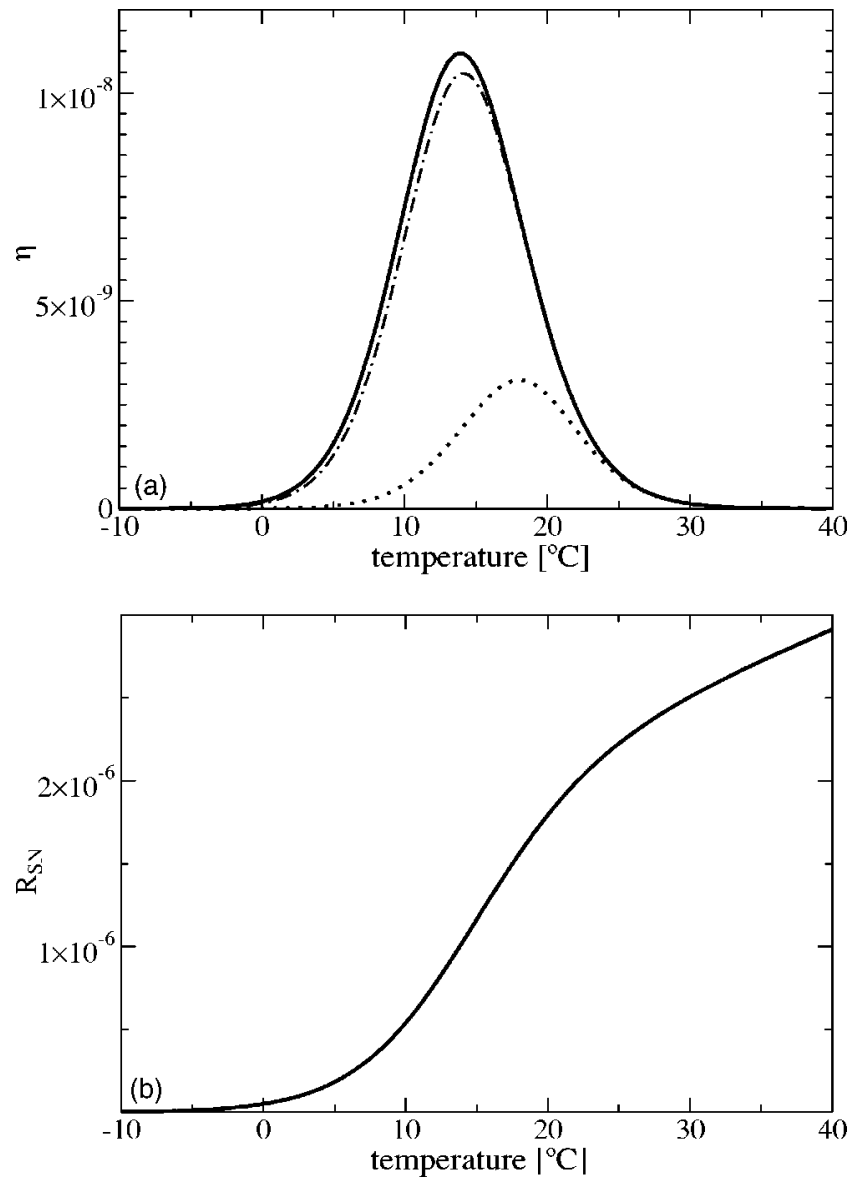

FIG. 3. Markovian three-state model of ion channel gating: (a) spectral power amplification $\eta$ [in units of $\left.(\Delta g q)^{2} /(\mathrm{J} / \mathrm{mol})^{2}\right]$ and (b) signal-to-noise ratio $R_{S N}$ [in units of $\pi(q A)^{2} /(\mathrm{J} / \mathrm{mol})^{2} \mathrm{~s}^{-1}$ ] versus temperature (in $\operatorname{deg} \mathrm{C}$ ) at different angular frequencies $\Omega$ of the harmonic signal. In (a), the full line corresponds to the adiabatic limit $\Omega \rightarrow 0$; the dash-dotted line corresponds to $\Omega=10 \mathrm{~s}^{-1}$ and the dotted line corresponds to $\Omega=100 \mathrm{~s}^{-1}$. The corresponding lines for $\Omega=0.1$ and $1 \mathrm{~s}^{-1}$ cannot be resolved from the zero-frequency limit. In (b), all the lines for the above mentioned four different frequencies merge with the zero-frequency result (solid line). Note that for the two-state Markovian counterpart of the considered three-state model with the rates depicted in Fig. 2(b), all the lines for the different frequencies merge with the zero-frequency result in (a). Likewise, in (b), the depicted line must be multiplied by the factor $1 / N(0) \approx 147.43$ to yield the corresponding Markovian two-state result.

parameters. This is so because a different value of $\nu_{0}$ would result in one and the same (unknown) systematic entropy correction for all metastable states. The entropy differences between the metastable states remain unchanged.

Our results for this choice of parameters are depicted in Fig. 3(a) for the spectral amplification $\eta$, and Fig. 3(b) for the corresponding signal-to-noise ratio $R_{S N}$. In particular, Fig. 3(a) convincingly demonstrates the possibility of stochastic resonance within the range of physiological temperatures when $\epsilon(T) \approx 0$, in accordance with Eq. (32). The SNR in Fig. 3(b) also exhibits the SR-like increase with increasing temperature. It does not, however, reach a resonance peak behavior in the corresponding temperature range. This peak behavior would formally be assumed [not depicted in Fig. 3(b)] only at physiologically unrealistic high temperatures of about $600{ }^{\circ} \mathrm{C}$. This resonance behavior is formally contained in Eq. (33) [notice the presence of $\nu(T)$ in the numerator there, which invalidates the criterion $\epsilon(T) \approx 0]$. The behavior at extreme high frequencies $\Omega \gg k_{i n}, k_{r}, k_{o}^{(0)}[\mathrm{cf}$. Eq. (41)] assumes qualitatively the same behavior as depicted in Fig. $3(\mathrm{~b})$; the only difference being that the SNR becomes increased by the factor $\left(C_{c^{\prime}}^{2}+1\right) / 2$. Surprisingly (in view of the rather large enthalpic barriers), this does not exclude the appearance of stochastic resonance in the SNR behavior at physiological temperatures at some intermediate high frequency of the signal. For the studied model system this occurs, for example, for $\Omega=10^{4} \mathrm{~s}^{-1}$ [this feature is not depicted because the accompanying spectral amplification $\eta(T)$ in Fig. 3(a) comes out to be extremely small].

\section{B. The case with a power law distribution}

To model the gating dynamics more realistically we next consider a description with a nontrivial nonexponential residence time distribution in the inactivated regime $\psi(\tau)$ in Eq. (28). In particular, we use a probability density $\psi(\tau)$ with the following characteristic function [29]:

$$
\tilde{\psi}(s)=\frac{1}{1+s\left\langle\tau_{r}\right\rangle g_{\alpha}\left(s \tau_{d}\right)},
$$

where

$$
g_{\alpha}(z)=\frac{\tanh \left(z^{\alpha / 2}\right)}{z^{\alpha / 2}} .
$$

The RTD (42) has been obtained in Ref. [29] as the solution of a conformational diffusion model accounting for an anomalous subdiffusion over energetically quasidegenerate substates within the given conformation, i.e., $I$ in the present work. This probability density possesses three parameters: the mean residence time $\left\langle\tau_{r}\right\rangle$ in this inactivated conformation, the conformational diffusion time $\tau_{d}$, and the index of subdiffusion $\alpha, 0<\alpha \leqslant 1$.

In spite of possessing three independent parameters only, the RTD (42) is capable of displaying a rich behavior. The case of single-exponential distribution with the rate parameter $k_{r}:==\left\langle\tau_{r}\right\rangle^{-1}$ is rendered for $\tau_{d}=0$. The case $\alpha=1$ corresponds to normal diffusion. For $\tau_{d} \ll\left\langle\tau_{r}\right\rangle$, the intraconformational diffusion effects are not essential. However, for $\tau_{d}$ $\gg\left\langle\tau_{r}\right\rangle$ and in the range $\left\langle\tau_{r}\right\rangle^{2} / \tau_{d} \ll \tau \ll \tau_{d}$ the corresponding RTD assumes a negative power law, i.e., $\psi(\tau) \propto \tau^{-3 / 2}$, which ends up in an exponential tail for $\tau>\tau_{d}$ [19]. For $\alpha<1$, the distribution (42) has an infinite variance, since $\left\langle\tau^{2}\right\rangle=\infty$ for any $\tau_{d} \neq 0$ and, depending on a subtle interplay of parameters, it can display up to three different power laws. These are $\psi(\tau) \propto \tau^{-\alpha / 2}$ initially, $\psi(\tau) \propto \tau^{-(2-\alpha / 2)}$ intermediately, and $\psi(\tau) \propto \tau^{-(2+\alpha)}$ asymptotically [29]. With the discussed choice of $\psi(\tau)$, the characteristic function of the RTD in the compound $C^{\prime}$ state reads 


$$
\widetilde{\psi}_{c^{\prime}}(s)=\frac{k_{o}^{(0)}\left[s g_{\alpha}\left(s \tau_{d}\right)+k_{r}\right]}{\left(s+k_{o}^{(0)}\right)\left[s g_{\alpha}\left(s \tau_{d}\right)+k_{r}\right]+k_{i n} s g_{\alpha}\left(s \tau_{d}\right)} .
$$

It possesses the same average residence time (36) and the coefficient of variation is

$$
C_{c^{\prime}}= \begin{cases}\infty, & \alpha<1, \\ \sqrt{1+\frac{2 k_{o}^{(0)} k_{i n}}{\left(k_{\text {in }}+k_{r}\right)^{2}}\left(1+k_{r} \tau_{d} / 3\right),} & \alpha=1 .\end{cases}
$$

Note that for $\alpha<1, C_{c^{\prime}}=\infty$. This implies that the detection of low frequency signals $\Omega \rightarrow 0$ is fully suppressed as $R_{S N}(\Omega)$ $\rightarrow 0$ at $\Omega \rightarrow 0[24,25]$. A bistable stochastic element with such properties can thus be used as a high pass filter for the signal transduction. In this case, the first leading terms of the small-s expansion of $\tilde{\psi}_{c^{\prime}}(s)$ read $\quad \tilde{\psi}_{c^{\prime}}(s) \approx 1-\left\langle\tau_{c^{\prime}}\right\rangle_{s}$ $+\frac{1}{3}\left(k_{i n} / k_{o}^{(0)} k_{r} \tau_{d}\right)\left(s \tau_{d}\right)^{1+\alpha}$. This corresponds asymptotically to a distribution $\psi_{c^{\prime}}(\tau) \propto \tau^{-(2+\alpha)}$, similar to the Pareto law behavior considered in Refs. [24,25].

With Eq. (42) in Eqs. (27), (28), and (30) one can evaluate the spectral amplification of the signal and the signal-tonoise ratio. We did this for the above set of thermodynamical parameters (see Table I) entering rates that are supplemented with the following parameters of the RTD in Eq. (42): $\alpha$ $=0.25, \tau_{d}=0.1 \mathrm{~s}$. As can be seen in Fig. 4(a), the nonMarkovian effects resolve the lines at the frequencies $\Omega=0.1$ and $1 \mathrm{~s}^{-1}$ which merge with the zero-frequency line in Fig. 3(a). Moreover, the different lines that merge in Fig. 3(b) become also resolved [see Fig. 4(b)], where upon increasing the angular frequency $\Omega$ the SNR $R_{S N}(\Omega, T)$ grows; namely, at zero frequency $R_{S N}(\Omega \rightarrow 0, T)=0$ and for $\Omega$ $\approx 100 \mathrm{~s}^{-1} R_{S N}(\Omega, T)$ reaches approximately (from below) its low frequency three-state Markovian limit in Fig. 3(b). With the further increase of $\Omega$ the SNR will, however, grow further, approaching asymptotically the two-state Markovian limit, where the non-Markovian form factor becomes unity, i.e., $N(\Omega)=1$. It is worth noticing that for the two lower curves in Fig. 4(b) the SNR seemingly saturates with increasing temperature. In fact, however, the SR behavior exhibits a rather broad maximum. The occurrence of this maximum is quite surprising (see the discussion at the end of Sec. IV A) and is due to non-Markovian effects.

Moreover, at variance with the SNR behavior (where the SNR increases with increasing angular frequency of signal) the corresponding SPA diminishes with increasing angular frequency. Therefore, there should exist an intermediate frequency range which would prove optimal for the detection of non-Markovian SR.

\section{SUMMARY}

With this work we have investigated stochastic resonance in a three-state non-Markovian model of ion channel gating. We note that our scheme of a three-state non-Markovian modeling is distinctly different from a similar one, recently applied to an excitable neuronal dynamics [38,39]. The latter model assumes a three-state system which cycles unidirectionally between three states, 1 (silent), 2 (excited), and 3
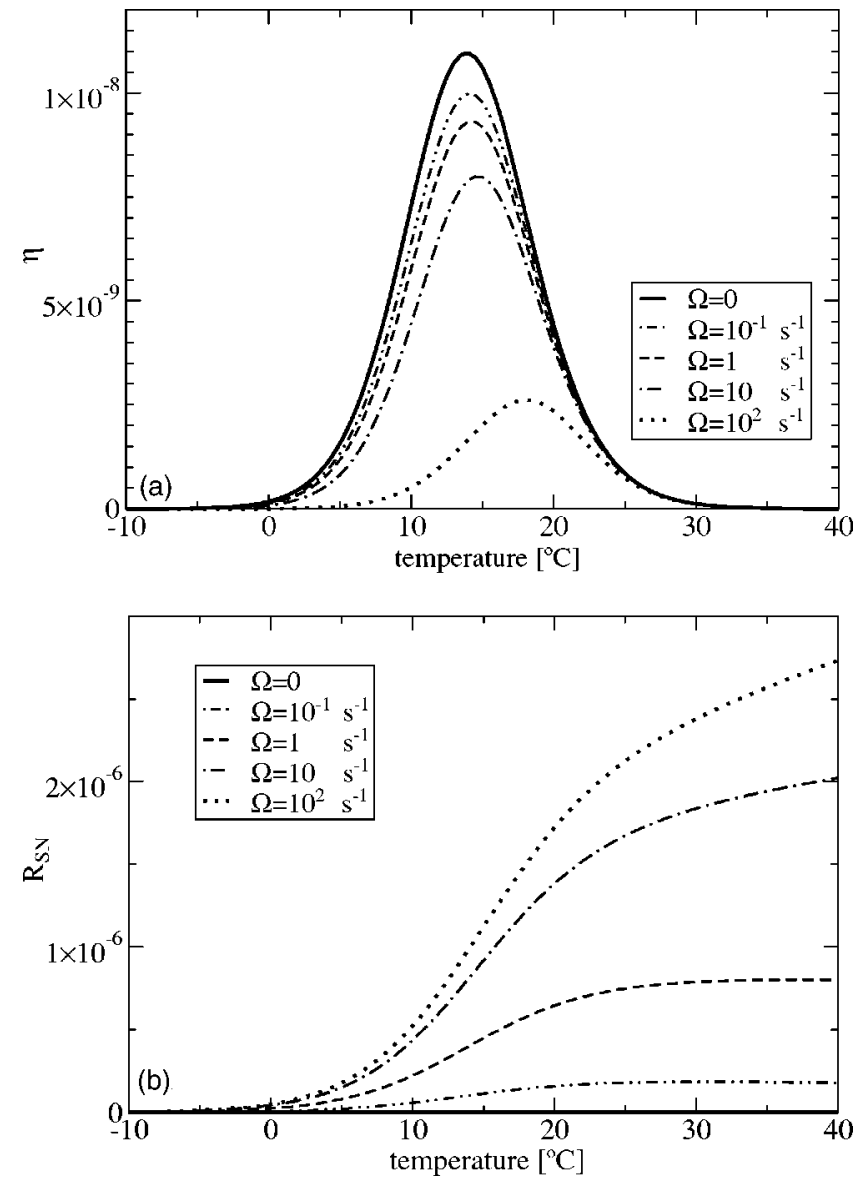

FIG. 4. The case with a power law distribution of residence times for three-state ion channel gating (cf. Fig. 1). Instead of a single-exponential RTD $\psi(\tau)$, the residence time distribution in the inactivated state is given now by Eq. (42) with $\alpha=0.25$ and $\tau_{d}$ $=0.1 \mathrm{~s}$. The other parameters remain the same as in Fig. 3. The manifest non-Markovian effects result in a resolution of the different lines that merged in Fig. 3. Note in (b) that the signal-to-noise ratio is fully suppressed toward zero for $\Omega \rightarrow 0$ (the result merges with the horizontal axis). The adiabatic limit of the corresponding Markovian three-state model is approximately assumed at $\Omega$ $=100 \mathrm{~s}^{-1}$; cf. the dotted line in (b) which compares with the solid line in Fig. 3(b).

(refractory), i.e., $1 \rightarrow 2 \rightarrow 3 \rightarrow 1$. This unidirectional cycling corresponds to processes that are very far from the thermal equilibrium and which require a continuous supply of free energy. In contrast, our modeling is compatible with the thermal equilibrium. The ion channel gating is commonly assumed to be a thermal equilibrium process [14]. This is in contrast to the situation with ion pumps and neuronal systems which do require a free energy supply for proper functioning. As has been shown in this work, our three-state description is compatible with the phenomenological two-state theory of non-Markovian stochastic resonance put forward in Ref. [24], while the nonequilibrium, three-state model of Ref. [39] is not within this class of system behaviors [25]. Nevertheless, the result of Ref. [39] for the spectral power amplification can be reproduced from our Eq. (A1) by specifying the RTD of the compound silent state (i.e., the silent state plus the refractory state) as a time convolution of two 
corresponding residence time probability densities and expanding it as in Eq. (A2). In other words, the model of Ref. [39] can be recovered as a special case within our general nonequilibrium approach put forward in Ref. [25] (Sec. IV therein).

In conclusion, the non-Markovian SR effects such as the suppression of the signal-to-noise ratio for low frequency signals and the resolution of different signal frequencies in the spectral amplification of signal in the presence of possibly large (entropic) asymmetries can be nicely modeled already within a three-state Markovian model. Such a threestate Markovian model yields the simplest non-Markovian model after the corresponding projection onto the subspace of two observable states. The signal-to-noise ratio remains, however, finite in the limit of the zero-frequency signal. Its complete suppression requires an infinite variance of the residence time intervals in the inactivated state. For such a manifest non-Markovian SR behavior, weak external oscillating signals with an intermediate frequency should be used in order to detect SR experimentally.

Our present results provide a theoretical proof for the occurrence of stochastic resonance in single biomolecules at physiological temperatures. This being so, our findings can guide the experimentalists to choose both the appropriate molecules for doing experiments and to identify the corresponding experimental parameter regime which in turn will reveal the SR phenomenon in a single ion channel. Presently there exist only very few experimental, ion-channel-based SR works. In Ref. [7] attempts to identify SR in a single ion channel were made; the occurrence of SR therein is, however, not convincing. In Ref. [6] one investigates SR and demonstrates SR. The phenomenon has been studied, however, only on the level of a small number of dynamically self-assembled alamethicin ion channels.

Our findings show that ideal candidates to observe SR experimentally on the level of single molecules are cold- (or heat-)sensitive ion channels [36]. From this viewpoint, we believe that a repetition of the experiments in Ref. [7] by resorting to such channels would indeed become successful for observing SR in a single ion channel operating within its physiological regime.

\section{ACKNOWLEDGMENTS}

This work has been in part supported by the Deutsche Forschungsgemeinschaft within SFB-486 "Manipulation of Matter on the Nanoscale," Project No. A10, and by the Ministry of Science and Education (Spain), Project No. FIS200402461. J.L.V. would like to thank all members of the Institute of Physics of the University of Augsburg for their kind hospitality during his stay in Germany.

\section{APPENDIX: DERIVATION OF LINEAR RESPONSE FUNCTION WITHIN NON-MARKOVIAN TWO-STATE THEORY}

In this appendix the result in Eq. (23) is rederived from the non-Markovian two-state theory of Ref. [25]. Namely, in Ref. [25] it has been shown that the linear response function of an arbitrary two-state renewal process characterized by the conditional RTDs $\psi_{j=1,2}(\tau \mid t)$ and the amplitude of conductance fluctuations $\Delta g$ to a periodic signal in Eq. (4) is given in the limit $A \rightarrow 0$ by

$$
\begin{aligned}
\tilde{\chi}(\Omega)= & -\frac{2 i \Delta g}{A \Omega} \frac{1}{\left\langle\tau_{1}\right\rangle+\left\langle\tau_{2}\right\rangle} \\
& \times \frac{\widetilde{\psi}_{2}^{(1)}(-i \Omega)\left[1-\widetilde{\psi}_{1}(-i \Omega)\right]-\widetilde{\psi}_{1}^{(1)}(-i \Omega)\left[1-\widetilde{\psi}_{2}(-i \Omega)\right]}{1-\widetilde{\psi}_{1}(-i \Omega) \widetilde{\psi}_{2}(-i \Omega)} .
\end{aligned}
$$

In Eq. (A1), $\widetilde{\psi}_{j}(s)$ denote the Laplace transforms of stationary RTDs in the absence of driving and $\widetilde{\psi}_{j}^{(1)}(s)$ are the Laplace transforms of the corresponding contributions in the expansion of the conditional, driven RTDs, i.e.,

$$
\psi_{j}(\tau \mid t)=\sum_{n=-\infty}^{\infty} \psi_{j}^{(n)}(\tau) \exp (-i n \Omega t)
$$

The quantities $\widetilde{\psi}_{j}^{(1)}(s)$ must be evaluated from an underlying multistate or continuous state dynamics to first order in $A$. Whenever $\widetilde{\psi}_{j}^{(1)}(s)$ satisfy the relation

$$
\widetilde{\psi}_{j}^{(1)}(-i \Omega)=-\frac{1}{2} \beta_{j} A\left[1-\psi_{j}(-i \Omega)\right],
$$

then the result of the phenomenological theory in Eq. (23) is recovered from Eq. (A1). For the exponential form of distribution of open residence times $\psi_{o}(\tau \mid t)=\psi_{2}(\tau \mid t)=k_{c}(t$ $+\tau) \exp \left[-\int_{t}^{t+\tau} k_{c}\left(\tau^{\prime}\right) d \tau^{\prime}\right]$, the relation (A3) is satisfied. We identify here the state " 1 " with the compound closed state " $(C, \mathrm{I})$ " and the state "2" with the open state " $O$ "; note also that $\beta_{1} \equiv \beta_{o}, \beta_{2} \equiv \beta_{c}$. This relation is valid as well for a multiexponential distribution which assumes a scaling relation among the rate parameters which is not modified by driving, i.e., a form-invariant RTD, cf. [25]. Below we demonstrate that the relation (A3) is also valid in the present case which obviously does not belong to the latter universality class. Nevertheless, the application of the phenomenological linear response theory to the considered non-Markovian processes [24] is justified.

In order to obtain the conditional survival probability of the compound closed state $\Phi_{1}(\tau \mid t):=\Phi_{1}(t+\tau, t)$ one needs to solve the system of equations

$$
\begin{gathered}
\frac{d}{d \tau} p_{c}(\tau \mid t)=-\left[k_{\text {in }}+k_{o}(t+\tau)\right] p_{c}(\tau \mid t) \\
+\int_{0}^{\tau} \Gamma\left(\tau-\tau^{\prime}\right) p_{I}\left(\tau^{\prime} \mid t\right) d \tau^{\prime}, \\
\frac{d}{d \tau} p_{I}(\tau \mid t)=k_{i n} p_{c}(\tau \mid t)-\int_{0}^{\tau} \Gamma\left(\tau-\tau^{\prime}\right) p_{I}\left(\tau^{\prime} \mid t\right) d \tau^{\prime},
\end{gathered}
$$

with the initial conditions $p_{c}(0 \mid t)=1$ and $p_{I}(0 \mid t)=0$. Then $\Phi_{1}(\tau \mid t)=p_{c}(\tau \mid t)+p_{I}(\tau \mid t)$. Asymptotically, the considered periodically driven renewal process becomes cyclic-stationary. For such a cyclic-stationary process, $\Phi_{1}(\tau \mid t)$ must be invari- 
ant under time shifts $t \rightarrow t+n T, n= \pm 1, \pm 2, \ldots$ with period $T=2 \pi / \Omega$; it thus can be expanded into Fourier series as in Eq. (A2). With $k_{o}(t)$ being a periodic function of time, we are seeking a solution of Eq. (A4) in the form $p_{\alpha}(\tau \mid t)$ $=\sum_{n=-\infty}^{\infty} p_{\alpha}^{(n)}(\tau) \exp (-i n \Omega t), p_{\alpha}^{(-n)}(\tau)=\left[p_{\alpha}^{(n)}(\tau)\right]^{*}, \alpha=c, I$. Invoking additionally a small-signal expansion (8) this yields an infinite system of coupled integro-differential equations for $p_{\alpha}^{(n)}(\tau)$ which upon the use of the Laplace-transform method results in a recurrent-difference relation for $\widetilde{p}_{c}^{(n)}(s)$, i.e.,

$$
\begin{gathered}
\left(s+k_{i n}+k_{o}^{(0)}-\frac{k_{i n} \widetilde{\Gamma}(s)}{s+\widetilde{\Gamma}(s)}\right) \widetilde{p}_{c}^{(n)}(s)+k_{o}^{(1)}\left[\widetilde{p}_{c}^{(n+1)}(s-i \Omega)\right. \\
\left.+\widetilde{p}_{c}^{(n-1)}(s+i \Omega)\right]=\delta_{n, 0},
\end{gathered}
$$

and a relation expressing $\widetilde{p}_{I}^{(n)}(s)$ through $\widetilde{p}_{c}^{(n)}(s)$, reading

$$
\widetilde{p}_{I}^{(n)}(s)=\frac{k_{i n}}{s+\widetilde{\Gamma}(s)} \widetilde{p}_{c}^{(n)}(s),
$$

where $k_{o}^{(1)} \propto A$ is given in Eq. (11). Note that $\widetilde{p}_{\alpha}^{(n)}(s)$ are nonlinear functions of the driving amplitude $A$. Equation (A5) can be solved perturbatively by using the corresponding expansions of $\widetilde{p}_{\alpha}^{(n)}(s)$ in $A$. The expansion of $p_{\alpha}^{(0)}(s)$ in $A$ starts from the driving-independent terms and obviously (from the symmetry reasons, $A \rightarrow-A$ ) does not contain a linear contribution in the amplitude strength $A$. Moreover, $\widetilde{p}_{\alpha}^{( \pm 1)}(s) \propto A$, to the lowest order in $A$. Hence, $\widetilde{p}_{\alpha}^{( \pm 2)}(s)=O\left(A^{2}\right)$ because the response at the second harmonic driving frequency cannot be linear. The use of standard perturbation theory then yields to the lowest order in $A$

$$
\begin{gathered}
\widetilde{p}_{c}^{(0)}(s)=\frac{1}{s+k_{i n}+k_{o}^{(0)}-k_{i n} \widetilde{\Gamma}(s) /[s+\widetilde{\Gamma}(s)]}+O\left(A^{2}\right), \\
\widetilde{p}_{c}^{(1)}(s)=-\frac{k_{o}^{(1)} \widetilde{p}_{c}^{(0)}(s+i \Omega)}{s+k_{i n}+k_{o}^{(0)}-k_{i n} \widetilde{\Gamma}(s) /[s+\widetilde{\Gamma}(s)]} .
\end{gathered}
$$

Equation (A7) determines in combination with Eq. (A6) for $n=0$ the stationary (i.e., in the absence of driving) survival function of the compound closed state in Eq. (18) and the corresponding RTD (19), respectively.

Equation (A8) yields in virtue of $\widetilde{p}_{c}^{(0)}(0)=1 / k_{o}^{(0)}+O\left(A^{2}\right)$ and Eq. (11) the result

$$
\begin{aligned}
\widetilde{p}_{c}^{(1)}(-i \Omega) & \\
\quad= & \frac{1}{2} A \frac{\beta_{o}}{-i \Omega+k_{i n}+k_{o}^{(0)}-k_{i n} \widetilde{\Gamma}(-i \Omega) /[-i \Omega+\widetilde{\Gamma}(-i \Omega)]} .
\end{aligned}
$$

Together with Eq. (A6) for $n=1$ this gives with $\beta_{o}=\beta_{1}$

$$
\widetilde{\Phi}_{1}^{(1)}(-i \Omega)=\frac{1}{2} A \beta_{1} \widetilde{\Phi}_{1}^{(0)}(-i \Omega) .
$$

Upon using the general relation, $\widetilde{\psi}_{\alpha}^{(n)}(s)=\delta_{n, 0}-s \widetilde{\Phi}_{\alpha}^{(n)}(s)$, Eq. (A10) thus yields the desired relation in (A3).
[1] L. Gammaitoni, P. Hänggi, P. Jung, and F. Marchesoni, Rev. Mod. Phys. 70, 223 (1998).

[2] V. S. Anishchenko, A. B. Neiman, F. Moss, and L. Schimansky-Geier, Phys. Usp. 42, 7 (1999) [Usp. Fiz. Nauk 169, 7 (1999)].

[3] K. Wiesenfeld and F. Moss, Nature (London) 373, 33 (1995).

[4] P. Hänggi, ChemPhysChem 3, 285 (2002).

[5] L. Callenbach, P. Hänggi, S. J. Linz, J. A. Freund, and L. Schimansky-Geier, Phys. Rev. E 65, 051110 (2002); J. A. Freund, L. Schimansky-Geier, and P. Hänggi, Chaos 13, 225 (2003); P. Talkner, L. Machura, M. Schindler, P. Hänggi, and J. Luczka, New J. Phys. 7, 14 (2005); J. Casado-Pascual, J. Gomez-Ordonez, M. Morillo, J. Lehmann, I. Goychuk, and P. Hänggi, Phys. Rev. E 71, 011101 (2005).

[6] S. M. Bezrukov and I. Vodyanoy, Nature (London) 378, 362 (1995); 385, 319 (1997).

[7] D. Petracchi, M. Pellegrini, M. Pellegrino, M. Barbi, and F. Moss, Biophys. J. 66, 1844 (1994).

[8] J. Galvanovskis and J. Sandblom, Biophys. J. 73, 3056 (1997).

[9] I. Goychuk and P. Hänggi, Phys. Rev. E 61, 4272 (2000).

[10] I. Goychuk, Phys. Rev. E 64, 021909 (2001).

[11] G. Schmid, I. Goychuk, and P. Hänggi, Europhys. Lett. 56, 22 (2001)

[12] P. Jung and J. W. Shuai, Europhys. Lett. 56, 29 (2001).

[13] G. Schmid, I. Goychuk, P. Hänggi, S. Zeng, and P. Jung, Fluct.
Noise Lett. 4, L33 (2004).

[14] B. Hille, Ionic Channels of Excitable Membranes, 3rd ed. (Sinauer Associates, Sunderland, MA, 2001).

[15] Single-Channel Recording, 2nd ed., edited by B. Sakmann and E. Neher (Plenum, New York, 1995).

[16] B. McNamara and K. Wiesenfeld, Phys. Rev. A 39, 4854 (1989); P. Jung and P. Hänggi, Europhys. Lett. 8, 505 (1989); Phys. Rev. A 44, 8032 (1991); 41, 2977 (1990).

[17] A. L. Hodgkin and A. F. Huxley, J. Physiol. (London) 117, 500 (1952).

[18] S. Marom, H. Salman, V. Lyakhov, and E. Braun, J. Membr. Biol. 154, 267 (1996).

[19] I. Goychuk and P. Hänggi, Proc. Natl. Acad. Sci. U.S.A. 99, 3552 (2002).

[20] I. Goychuk and P. Hänggi, Physica A 325, 9 (2003).

[21] L. S. Liebovitch, J. Fishbarg, and J. P. Koniarek, Math. Biosci. 84, 37 (1987); L. S. Liebovitch and J. M. Sullivan, Biophys. J. 52, 979 (1987).

[22] B. J. West and W. Deering, Phys. Rep. 246, 1 (1994).

[23] S. Marom, J. Membr. Biol. 161, 105 (1998).

[24] I. Goychuk and P. Hänggi, Phys. Rev. Lett. 91, 070601 (2003).

[25] I. Goychuk and P. Hänggi, Phys. Rev. E 69, 021104 (2004).

[26] G. L. Millhauser, E. E. Salpeter, and R. E. Oswald, Proc. Natl. Acad. Sci. U.S.A. 85, 1503 (1988).

[27] C. A. Condat and J. Jäckle, Biophys. J. 55, 915 (1989). 
[28] F. Bezanilla, E. Perozo, and E. Stefani, Biophys. J. 66, 1011 (1994).

[29] I. Goychuk and P. Hänggi, Phys. Rev. E 70, 051915 (2004).

[30] V. M. Kenkre, E. W. Montroll, and M. F. Shlesinger, J. Stat. Phys. 9, 45 (1973).

[31] A. I. Burshtein, A. A. Zharikov, and S. I. Temkin, Theor. Math. Phys. 66, 166 (1986).

[32] B. D. Hughes, Random Walks and Random Environments (Clarendon Press, Oxford, 1995), Vol. 1.

[33] I. Goychuk, Phys. Rev. E 70, 016109 (2004).

[34] D. R. Cox, Renewal Theory (Methuen, London, 1962).
[35] R. L. Stratonovich, Topics in the Theory of Random Noise (Gordon and Breach, NewYork, 1963), Vol. 1, p. 176.

[36] T. Voets, G. Droogmans, U. Wissenbach, A. Janssens, V. Flockerzi, and B. Nilius, Nature (London) 430, 748 (2004).

[37] P. Hänggi, P. Talkner, and M. Borkovec, Rev. Mod. Phys. 62, 251 (1990).

[38] B. Linder, J. Garcia-Ojalvo, A. Neiman, and L. SchimanskyGeier, Phys. Rep. 392, 321 (2004).

[39] T. Prager and L. Schimansky-Geier, Phys. Rev. Lett. 91, 230601 (2003) 